\title{
Revisiting a GWAS peak in Arabidopsis thaliana reveals possible confounding by genetic heterogeneity
}

\author{
Eriko Sasaki ${ }^{1,3}$, Thomas Köcher $^{2}$, Danièle L Filiault ${ }^{1}$, Magnus Nordborg ${ }^{1^{*}}$ \\ 1. Gregor Mendel Institute, Austrian Academy of Sciences, Vienna BioCenter (VBC), Dr. \\ Bohr-Gasse 3, 1030 Vienna, Austria \\ 2. Vienna BioCenter Core Facilities, Vienna BioCenter (VBC), Dr. Bohr-Gasse 3, 1030 \\ Vienna, Austria \\ 3. Faculty of Science. Kyushu University 744, Motooka, Nishi-ku, Fukuoka 819-0395, Japan \\ * Corresponding author: Magnus Nordborg nordborg@gmi.oeaw.ac.at
}

\begin{abstract}
Genome-wide association studies (GWAS) have become a standard approach for exploring the genetic basis of phenotypic variation. However, correlation is not causation, and only a tiny fraction of all associations have been experimentally confirmed. One practical problem is that a peak of association does not always pinpoint a causal gene, but may instead be tagging multiple causal variants. In this study, we reanalyze a previously reported peak associated with flowering time traits in Swedish in Arabidopsis thaliana. The peak appeared to pinpoint the AOP2/AOP3 cluster of glucosinolate biosynthesis genes, which is known to be responsible for natural variation in herbivore resistance. Here we propose an alternative hypothesis, by demonstrating that the AOP2/AOP3 flowering association can be wholly accounted for by allelic variation in two flanking genes with clear roles in regulating flowering: $N D X 1$, a regulator of the main flowering time controller FLC, and GA1, which plays a central role in gibberellin synthesis and is required for flowering under some conditions. In other words, we propose that the AOP2/AOP3 flowering-time association is yet another example of a spurious, "synthetic" association, arising from trying to fit a single-locus model in the presence of two statistically associated causative loci.
\end{abstract}

\section{Introduction}

Genome-wide association studies (GWAS) have become an essential tool for studying the genetics of natural variation. In addition to its tremendous impact on human genetics, GWAS is being applied routinely to a wide range of species, and massive numbers of genotype-phenotype associations have been revealed (Atwell et al., 2010; Flint and Eskin, 2012; MacArthur et al., 2017). However, only for a tiny subset do we have any idea of why the association exists, i.e., the molecular mechanism. There are many reasons for this, but an important one is that peaks do not always pinpoint the causal genes (Hormozdiari et al., 2014; Tam et al., 2019). In settings where the environment cannot be controlled, spurious associations may simply arise because of environmental confounding, but our focus here is on confounding by the genetic background, and in particular on genetic background effects that are not sufficiently diffuse to be readily be removed by approximate methods like kinship or Principal Component corrections (Atwell et al., 2010; Vilhjálmsson and Nordborg, 2013). Such effects may arise whenever there is linkage disequilibrium between non-trivial allelic effects (Platt et al., 2010; Dickson et al., 2010), and is a well-known problem for 
fine-mapping when there is allelic heterogeneity. In Arabidopsis, examples include multiple functional alleles of FRIGIDA (FRI) (Atwell et al., 2010), and DELAY OF GERMINATION1 (DOG1) (Kerdaffrec et al., 2016). Less clear is how frequently spurious associations arise from genetic heterogeneity, i.e., from alleles for different genes affecting the same trait. In this paper we discuss what we believe to be an example of this: causal allelic variation at two different genes, separated by roughly $120 \mathrm{~kb}$, inducing a spurious peak of association in a third gene located between the causal loci.

\section{Results}

\section{GWAS for flowering time suggested a role for AOP2}

Flowering time is an adaptively and agriculturally important trait that has been intensively studied in Arabidopsis thaliana. Thanks to decades of functional work, the pathways involved in flowering time regulation (and their interaction with the environment) are extremely well understood (Koornneef et al., 1998; Srikanth and Schmid, 2011; Andrés and Coupland, 2012). Known flowering-time regulators are also highly variable in nature, and GWAS for flowering time typical reveal a variety of known loci (Brachi et al., 2010; Atwell et al., 2010; Li et al., 2010; Sasaki et al., 2015; 1001 Genomes Consortium, 2016; Zan and Carlborg, 2019)

A major source of natural variation for flowering is a variety of loss-of-function alleles of FRI, which regulates FLOWERING LOCUS C (FLC), a key regulator of flowering time, crucial for helping plants flower in the right season by "remembering" exposure to cold winter temperatures using a fascinating epigenetic mechanism (Whittaker and Dean, 2017). Indeed, FRI was first identified through natural variation (Johanson et al., 2000), but, despite explaining a considerable fraction of the variation for flowering time, has proven difficult to map using GWAS, mostly because it has such high allelic heterogeneity (Atwell et al., 2010). However, several GWAS identified a strong peak roughly $1 \mathrm{Mb}$ from $F R I$, a peak which stood out because it was not obviously associated with a known flowering time gene (Brachi et al., 2010; Atwell et al., 2010; Li et al., 2010; Zan and Carlborg, 2019). Instead, this peak appeared to pinpoint the highly variable and adaptively important ALKENYL HYDROXALKYL PRODUCING (AOP) cluster (Atwell et al., 2010; Kerwin et al., 2011; Katz et al., 2020), containing three tandemly duplicated genes involved in the synthesis of glucosinolates, secondary metabolites that play important role in defense against herbivory (Kliebenstein et al., 2001). In the Swedish population, the strongest association was found for the SNP at chr4:1355057, 961 bp upstream of $A O P 2$, and strongly correlated both with flowering time and FLC expression (Figs. 1 and S1). Here we focus on this association, and return to associations in other populations in the discussion. 

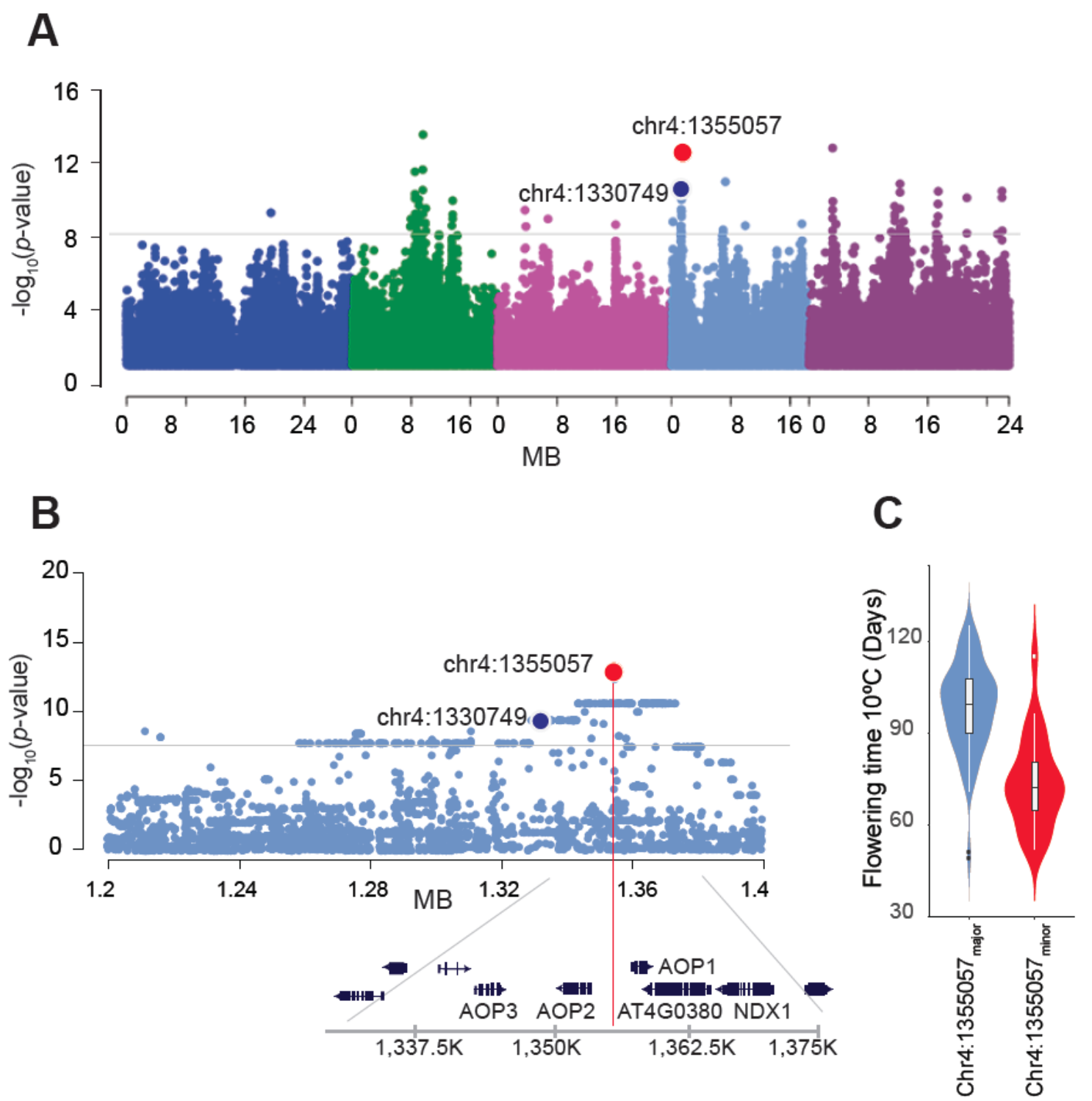

Fig. 1 GWAS for flowering time revealed a peak centered on the chromosome 4 AOP cluster. (A) Genome-wide Manhattan plot for flowering time at $10^{\circ} \mathrm{C}$ in 132 Swedish lines, with SNP reported in Atwell et al. (2010) highlighted in blue (chr4: 1330749), and the strongest association in red (chr4: 1355057). A linear model without structure correction was used (see Methods). (B) Zoom-in on the peak, with gene annotation. (C) Violin plot showing the difference in flowering time between lines carrying major and minor alleles at chr4:1355057.

\section{Functional variation at $A O P 2$ does not affect flowering time}

Although a transgene experiment had shown that AOP2 (but not AOP3) could affect flowering time (Kerwin et al., 2011; Jensen et al., 2015), pleiotropic effects on flowering time are common (Chong and Stinchcombe, 2019), and we were not convinced that this was the explanation for the $A O P 2$ peak. To investigate this further, we first explored the functional AOP2 variants tagged by chr4:1355057. Jensen et al. (2015) showed that A. lyrata AOP2 delays flowering when overexpressed in the reference line Col-0, which carries a natural non-functional $A O P 2$ allele due to a 5-bp deletion causing a frameshift and leading to accumulation of different glucosinolates (Kliebenstein et al., 2001). They suggested that delayed flowering results from an interaction between the glucosinolate and flowering 
pathways. Based on these results, functional $A O P 2$ alleles should be associated with delayed flowering in $A$. thaliana. AOP2 has multiple alleles inducing frameshift in addition to the Col-0 allele (Neal et al., 2010). In the Swedish population, five indels that could induce frameshift were identified, including the Col-0 type (Fig. 2A; Table S1).

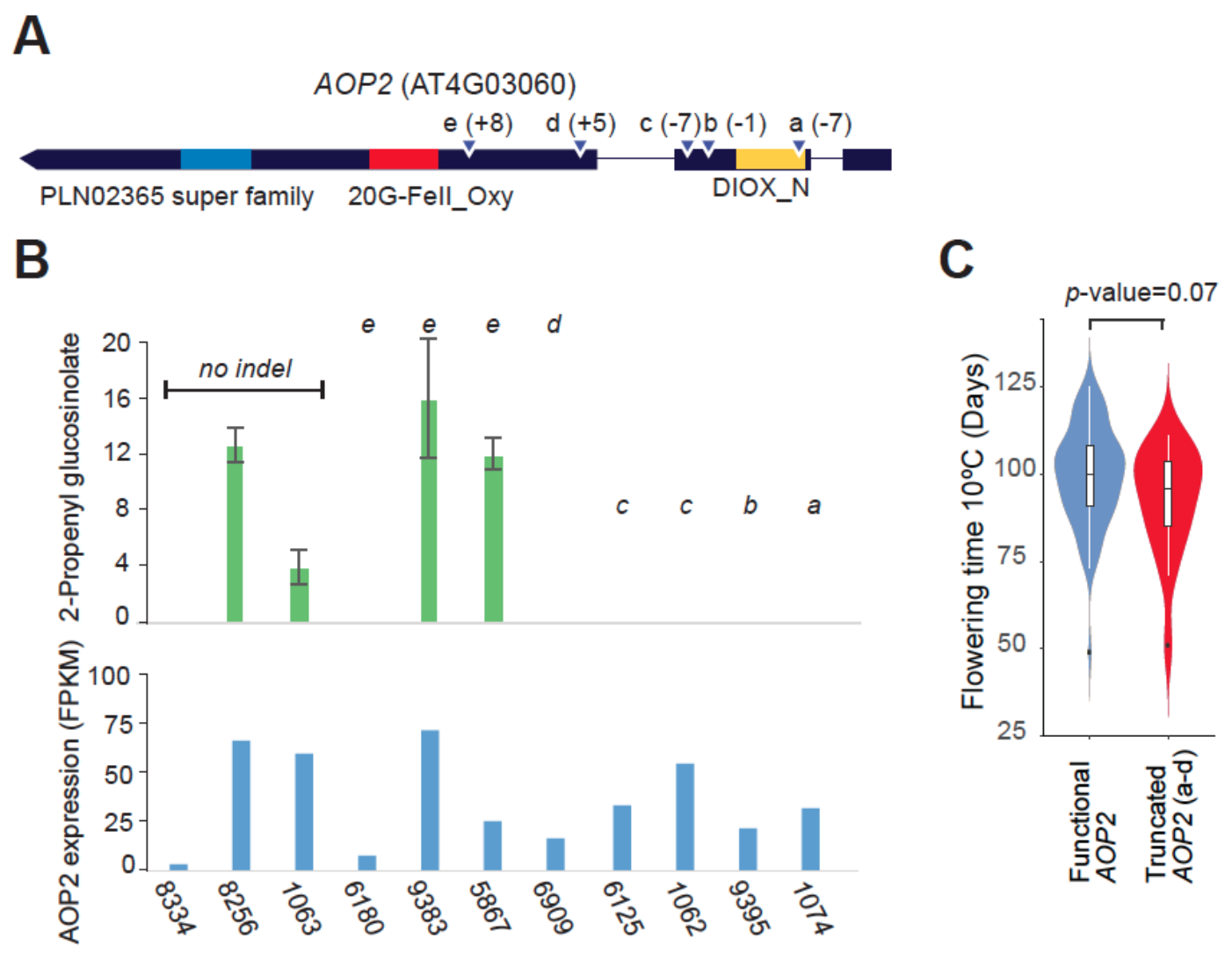

Fig. 2 Functional validation of AOP2 alleles. (A) The gene model of AOP2.1. Colored parts are conserved protein domains. Triangles show predicted indels that could cause frameshift. + and - are the indel size from the reference sequence. (B) Accumulation of 2-propenyl glucosinolate in each type of allele and the AOP2 expression. Marks from a to e are corresponding to the indels. (C) The flowering time based on $A O P 2$ indel genotypes. Functional $A O P 2$ is the genotype without frameshift and truncated AOP2 has frameshift (a-d). Only chr4:1355057 reference lines with expressed AOP2 were used for the plots to control for the effect of chr4:1355057 (which is of course known to be associated with flowering time variation).

We assessed the functional effect of these five indels directly using mass spectrometry (Fig. 2B). AOP2 converts 3-methylsulfinylpropyl and 4-methylsulfinylbutyl glucosinolate to 2-propenyl and 3-butenyl glucosinolate, respectively (Kliebenstein et al., 2001). Lines having any indel in the second exon (a to $\mathrm{c}$ in Fig. 2A) or the Col-0 indel (d) in the third exon did not accumulate 2-propenyl and 3-butenyl glucosinolate, although transcripts were detected in all cases (Fig. 2B; Table S2). The fifth insertion (e) did not appear to affect 2-propenyl and 3-butenyl glucosinolate accumulation significantly.

Contrary to a causal role for glucosinolate pathways in flowering time variation, AOP2 functionality is neither correlated with chr4:1355057 nor flowering time. For example, all 105 
lines carrying the reference allele at chr4:1355057 show substantial AOP2 expression, and 19 lines of them have indels that disrupt function (Table S1), but this has no significant effect on flowering time (Fig. 2C). There is a weak correlation between AOP2 expression and flowering (Fig. S1B), but it seems unlikely that this relationship reflects causality, when functional allelic variation does not. In conclusion, although it has been demonstrated that AOP2 can affect flowering time (Kerwin et al., 2011; Jensen et al., 2015), the considerable functional $A O P 2$ variation observed in the Swedish population is not strongly correlated with flowering time, suggesting that the major association between chr4:1355057 and flowering time arises for other reasons.

\section{The AOP2 peak tags a diverged allele of NDX1}

In order to identify potential causal variants, we dissected the local haplotype structure surrounding chr4:1355057 using principal component analysis (PCA) (Fig. 3A). Consistent with the fact that the Swedish population has a strong north-south population structure (Long et al., 2013), the first two principal components with the latitude of origin (PC1 $r^{2}=0.28$; PC2 $\left.r^{2}=0.25\right)$. However, the third principal component was not correlated with global structure, but rather identified an extended haplotype carried by 20 of the 51 lines that also carried the non-reference chr4:1355057 allele (Fig. 3A). This haplotype (denoted chr4:1355057b) contained three genes upstream of AOP2, including NDX1 (AT4G03090; chr4:1366053..1371237) - a known regulator of FLC that binds to the promoter region of COOLAIR, the antisense transcript of $F L C$, and inhibits the degradation of $F L C$ by stabilizing the R-loop (Sun et al., 2013) (Fig. 3B).

Furthermore, the chr4:1355057b haplotype is perfectly associated with a highly diverged NDX1 allele (Fig. 3C). The non-synonymous sequence divergence between this allele and the reference allele is close to $1 \%$, and the changes are predicted to affect gene activity (Sun et al., 2013) (Fig. S2). Mutant lines confirmed that NDX1, unlike neighboring genes, has a significant effect on flowering (Fig. S3). 
A
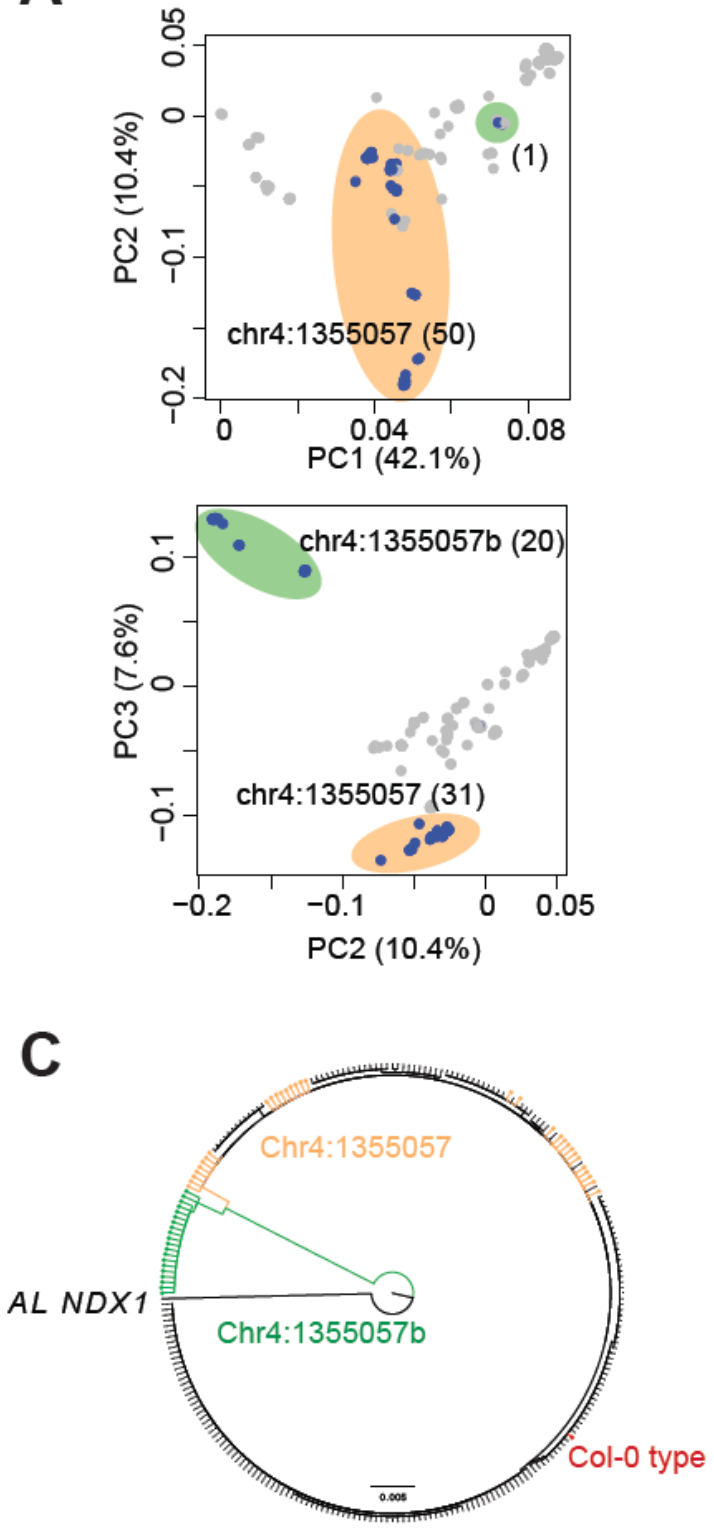

B

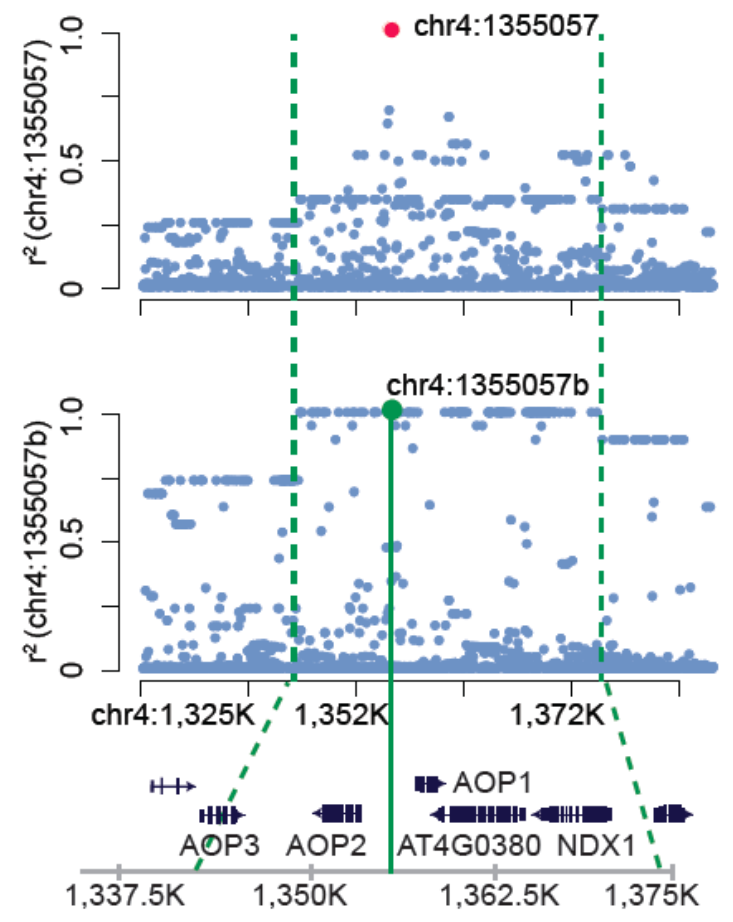

Fig. 3 Haplotype structure around the AOP peak. (A) PCA of SNPs in the $60 \mathrm{~kb}$ region around chr4:1355057. Major (reference type) and minor alleles of chr4:1355057 are plotted in grey and blue, respectively. The clusters of minor chr4:1355057 alleles are indicated in orange and green, respectively. Numbers in brackets are allele counts. (B) Decay of linkage disequilibrium with respect to chr4:1355057 and chr4:1355057b, the subset of the chr4:1355057 minor alleles associated with an extended haplotypes (the borders of which are indicated by vertical green lines) (C) Neighbor-joining tree of NDX1 alleles in the Swedish population $(n=259)$. Diverged NDX1 haplotypes associated with the chr4:1355057b haplotype are shown in green, while orange denotes remaining lines associated with rest of the chr4:1355057 minor allele.

\section{Multilocus GWAS including NDX1 reveals a new association near GA1}

These observations suggested that the flowering time association peak centered on chr4:1355057 could partly be due allelic variation at NDX1 (Fig. 4A). To explore this further, 
we performed GWAS while including the chr4:1355057b haplotype as a cofactor to regress out the effect of the NDX1 polymorphism (Fig. 4B). Doing so did not eliminate the significant peak on chromosome 4 (suggesting that NDX1 is not the only causal variant), but moved it over $100 \mathrm{Kbp}$ in the opposite direction of NDX1. The "new" peak was quite broad and flat, but the second strongest association (chr4:1236543; - $\log _{10} p$-value=11.85; MAC=15) was 1.1 Kbp downstream of another well-known flowering regulator, GIBBERELLIC ACID REQUIRING 1 (GA1 Chr4:1237671..1244822) that is essential for gibberellic acid biosynthesis (Sun and Kamiya, 1994). Gibberellin plays a crucial role in the transition to flowering through regulation of LEAFY (LFY) and FLOWERING TIME LOCUS T (FT) (Blazquez et al., 1998; Porri et al., 2012), and loss of function mutants of GA1 cannot flower under certain conditions (Reeves and Coupland, 2001).

Previous GWAS and linkage mapping studies have suggested that allelic variation at GA1 plays a role in flowering time variation, and the association is known to be sensitive to population structure correction (Brachi et al., 2010). These kinds of problems are often caused by extensive linkage disequilibrium, the existence of which is evident (Fig. S4). This can also be seen by carrying out a third GWAS, now with the chr4:1236543 (GA1) SNP as a cofactor, because this causes an increase in the height of the $A O P$ peak demonstrating that these peaks are indeed not independent (Fig. 4C).

\section{Polymorphisms at GA1 and NDX1 jointly explain the $A O P$ association}

Finally, we asked whether allelic variants at GA1 and NDX1 were jointly sufficient to explain the peak centered on AOP2. When we performed GWAS using both chr4:1236543 (GA1) and chr4:1355057b (NDX1) as cofactors, the peak at the $A O P$ cluster completely disappeared (Fig. 4D) - just as if we had taken chr4:1355057 (AOP2) as a cofactor (Fig. S5). The distribution of phenotypes explained by chr4:1355057 is consistent with a cumulative contribution by rarer alleles at chr4:1355057b and chr4:1236543 (Fig. 4D), and explain more of the variation (as expected given the extra parameter). The same pattern was seen in GWAS for FLC expression (Figs. S5 and 6). These results suggest that the major flowering time association at the $A O P$ cluster may be a spurious, "synthetic" association that results from the complex pattern of linkage disequilibrium between causal polymorphism at two nearby loci, GA1 and NDX1. The existence of extensive linkage disequilibrium (Fig. S4) and haplotype structure (Fig. S7) in the region is clear, although we note the average decay of linkage disequilibrium is by no means unusual relative to the rest of the genome (Fig. S8). 


\section{A}
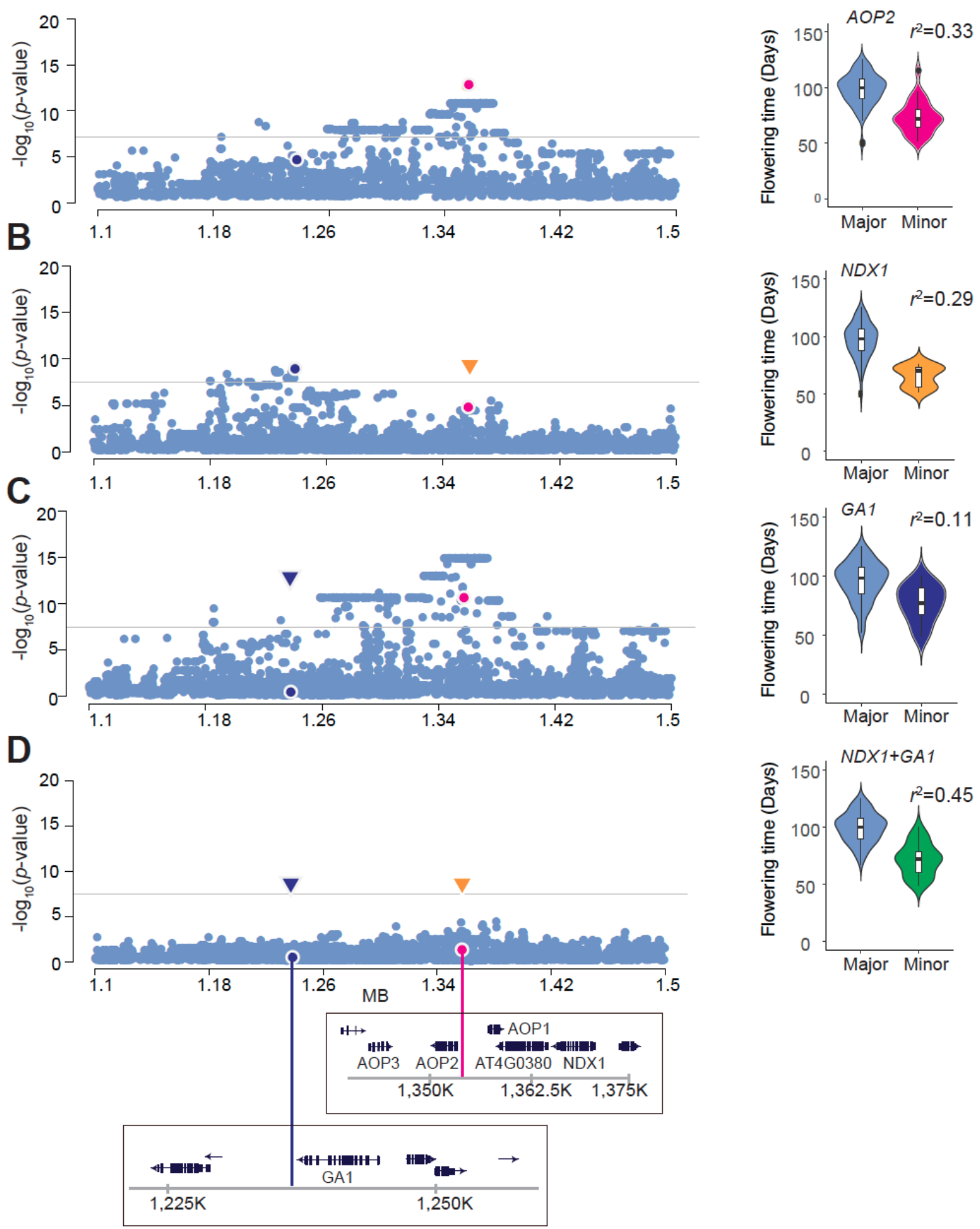

Fig. 4 Conditional GWAS suggests genetic heterogeneity. Zoom-in plots of conditional GWAS surrounding chr4:1355057 with violin plots illustrating how much of the variation is explained by each model. Arrows on the Manhattan plots indicate SNPs used for the cofactors. (A) The original association identified an association in AOP2 (chr4:1355057, magenta) that explained $33 \%$ of the flowering time variation. (B) Conditional GWAS using a diverged NDX1 haplotype (chr4:1355057b, orange) that explained $29 \%$ of the variation revealed an association near GA1 (chr4:1236543, dark blue). (C) Conditional GWAS using GA1 peak that explained $11 \%$ of the variation. (D) Conditional GWAS using both NDX1 and GA1 as co-factors fully explained the original peak (explaining $45 \%$ of the variation). 


\section{Discussion}

In this paper we have presented an alternative interpretation for a published GWAS peak, a potential example of genetic confounding. We demonstrate that a reproducible association between flowering time (and FLC expression) and SNPs in the AOP2/AOP3 glucosinolate biosynthesis cluster can alternatively be explained using a two-locus model, where the causal variants are in two flanking genes directly involved in the regulation of flowering: GA1, essential for gibberellin synthesis, and $N D X 1$, a regulator of $F L C$. Under this interpretation, the $A O P 2$ peak is a spurious association (Figure 5), an artefact of incorrectly fitting a single-locus model in the presence of two causative loci (Platt et al., 2010; Dickson et al., 2010; Atwell et al., 2010). The problem is analogous to the problem of "ghost QTLs" in classical linkage mapping (Haley and Knott, 1992; Martínez and Curnow, 1992).

Two-locus model

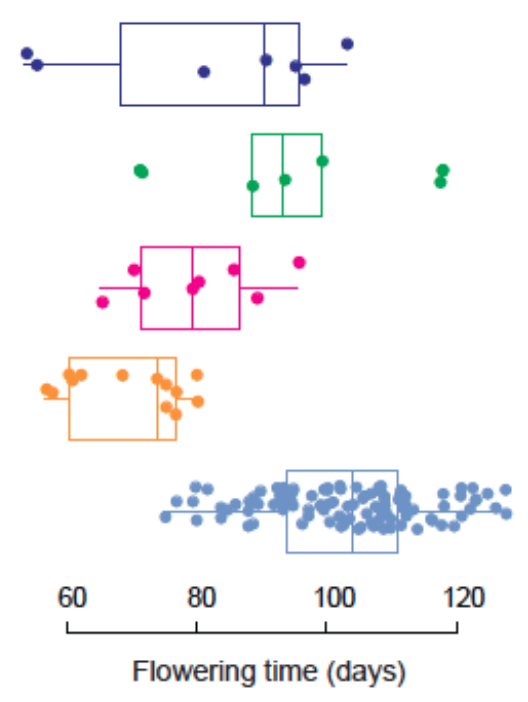

\section{GA1 AOP2 NDX1}
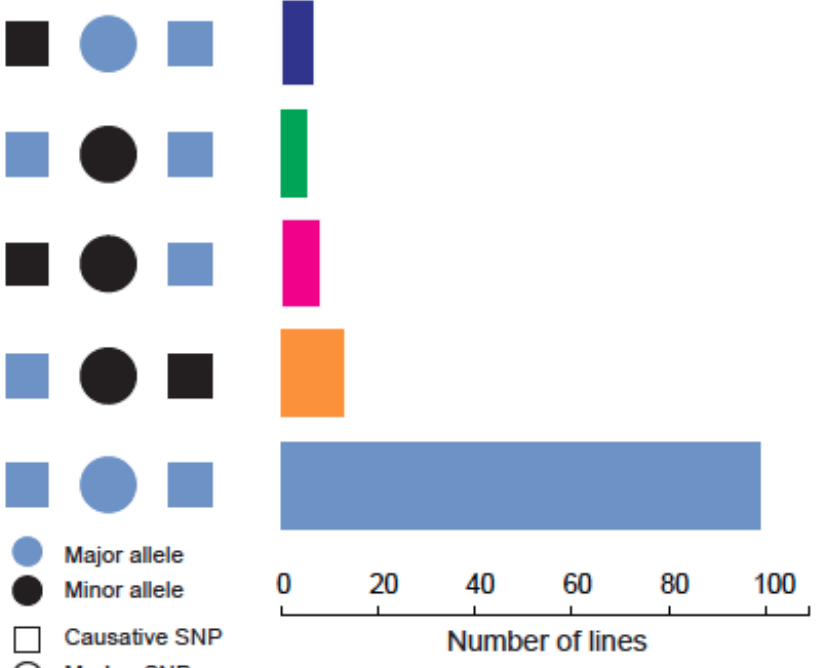

Single-locus model
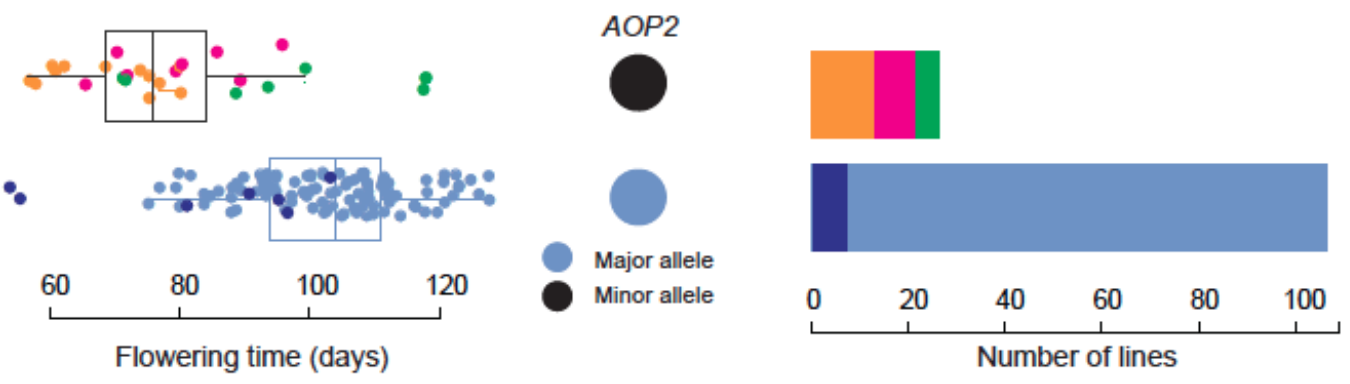

Fig. 5 Summary of our results. We consider three di-allelic SNPs, in (or near) GA1, AOP2, and $N D X 1$, respectively. Because of strong linkage disequilibrium, the minor NDX1 allele is only found on haplotypes with the major GA1 and minor AOP2 allele, which means there are only five haplotypes three are missing. The figure shows the flowering time distribution and observed frequency for each of these haplotypes. Our model is that the minor GA1 and NDX1 alleles are tagging early-flowering alleles at these two loci, and that the minor AOP2 allele is associated with early flowering because it is the best single locus that tags both of these loci. Colors in the single-locus model correspond to those in the two-locus model. 
We emphasize that neither of these two models has been experimentally confirmed. Merely knocking genes out is not sufficient for a trait like flowering time, which has been shown to be highly "omnigenic" (Boyle et al., 2017) in the sense that random knock-out mutations are as likely as a priori candidates to affect it (Chong and Stinchcombe, 2019). Because direct gene replacement is not feasible in $A$. thaliana, experimental testing of these two models would thus entail knocking out the native allele at multiple loci in multiple genetic backgrounds, and replacing it with cloned native alleles, using on the order of 50 independent transgenic lines per construct to account for position effects (Li et al., 2014). In late-flowering $A$. thaliana, with an essentially annual life-cycle, this would be a multi-year project requiring considerable resources.

That said, we believe that our two-locus model (involving two known flowering regulators) is a more likely explanation for the association seen here than the single-locus model (involving glucosinolate production). We say this primarily because, although flowering time is clearly "omnigenic" in the sense of presenting a large mutation target, GWAS results for flowering time (like many other phenotypes in A. thaliana) have generally showed a strong over-representation for genes in known pathways (Atwell et al., 2010; Sasaki et al., 2015). This is in sharp contrast to human genetics, where GWAS results have generally been extremely difficult to interpret (Boyle et al., 2017). A plausible explanation for this difference is that much of the variation in A. thaliana is adaptive (Atwell et al., 2010).

Indeed it may well be the case that selection is indirectly responsible for the $A O P 2$ flowering time association. The role of the $A O P$ cluster in defense against herbivory is well established (Kliebenstein et al., 2001), and it is tempting to speculate that strong selection on glucosinolate variation could have contributed to the complex haplotype structure in the region - leading to associations between SNPs in AOP2 and functional variants in nearby flowering regulators simply through random hitchhiking (Maynard Smith and Haigh, 1974). It should be noted that while a two-locus model appears to be required to explain the AOP2 flowering-time association seen in the Swedish population, the association seen in two other samples (Atwell et al., 2010; Li et al., 2010), can simply be explained by extremely strong linkage disequilibrium between AOP2 variants and the diverged NDX1 haplotype described above (Figure S9). Spurious flowering time associations due to regional selection on other traits has also been suggested for maize (Larsson et al., 2013).

To conclude, while we may never know which (if any) of the models proposed here is correct, there is no doubt that spurious associations like this do exist, and may complicate interpretation of mapping results (Huang et al., 2010; Atwell et al., 2010; Larsson et al., 2013; Hormozdiari et al., 2014; Kerdaffrec et al., 2016). Although representing a difficult model-selection problem, better methods for systematically identifying such associations could be a very cost-efficient way of getting more information out of GWAS results.

\section{Materials and methods}

\section{Data sets}

We used published $A$. thaliana data sets containing genotypes (Long et al., 2013), RNA-seq transcriptome data (Dubin et al., 2015), as well as flowering time phenotypes (Sasaki et al., $2015,2017)$ for the Swedish population. All plants were grown under a constant $10^{\circ} \mathrm{C}(132$ lines) in $16 \mathrm{~h}$ day length condition. For RNA seq analysis, RNA was extracted from whole rosettes collected at 11-12 $\mathrm{h}$ after dawn at nine-leaf stage (Dubin et al., 2015). Other phenotype data, including FLC expression (Atwell et al., 2010) and flowering time under 
Sweden spring condition in 2008 (Li et al., 2010), were obtained from AraPheno (Seren et al., 2017).

\section{Plant materials}

Loss-of function mutants of AT4G03080 (SALK_051383), AT4G03100 (SALK_082878), and NDX1 (WiscDsLox344A04) (Sun et al., 2013) were grown with the wild type under a constant $21^{\circ} \mathrm{C}$ in $16 \mathrm{~h}$ day length condition.

\section{Statistical analysis}

\section{GWAS}

GWAS was performed using LIMIX version 3.0.4 (Lippert et al., 2014) with full genome SNPs in a Swedish population ( $n=132$ ) (Long et al., 2013) and 250K SNP chip genotypes in RegMap panel (Horton et al., 2012). A linear mixed model (LMM) was used to correct population structure with a kinship matrix representing genetic relatedness (IBS) (Yu et al., 2006; Kang et al., 2008). For GWAS without correction of population structure (Figs 1, 4, S1, S5, and S6), a linear regression model was used. Uncorrected GWAS was used because several of the variants in the chromosome 4 region are strongly correlated with population structure in the Swedish population, rendering fine-mapping impossible because of lack of power. Note that our primary interest is not the genome-wide significance of the region, but rather identifying potential causal SNPs within it. It has previously been observed that kinship correction can obscure causality locally (Kerdaffrec et al., 2016). Cofactor analysis was performed using a multiple linear regression model without population structure correction in the Swedish population (Figs 4, S1, S4, and S5), and LMM for global population (Fig S9).

\section{PCA}

The entire Swedish population (259 lines (Long et al., 2013)) were used to analyze local genetic structure around the AOP cluster. SNPs in the $60 \mathrm{~kb}$ region around chr4:1355057 were extracted and analyzed using the prcomp function in R (https://www.r-project.org).

\section{Haplotype analysis}

For the analysis, SNPs covering GA1 and NDX1 regions, including the $30 \mathrm{Kbp}$ upstream of GA1 and downstream of NDX1, were used from a pre-imputation version of the Regional Mapping Project SNP panel, including 1307 global lines (Horton et al., 2012). These SNPs were used as the input into fastPHASE version 1.4.8 (Scheet and Stephens, 2006), which was run using the default settings as described in (Li et al., 2014).

\section{Genotyping NDX1 and AOP2}

For population samples, amino acid sequences of NDX1 and AOP2 were predicted using genome data, including SNPs and short indels (Long et al., 2013). NDX1 sequences of Col-0 and chr4:1355057b alleles were also confirmed by Sanger sequencing after cloning the 7.8 Kbp region with forward primer 5'-CTGGTAAATACTGTGTGTAGACAATTCT-3' and reverse primer 5'-TCGATGTTTGACGGCAAAGGATGAAG-3'. Line 6180 (TDÄL 07; latitude 62.6322 longitude 17.6906) was chosen to represent chr4:1355057b alleles. All predicted chr4:1355057b allele-specific SNPs were confirmed by the Sanger sequencing.

\section{Measurement of expression levels}

For population samples, $F L C$ and $A O P 2$ expressions were extracted from RNA-seq data of leaf tissue under $10^{\circ} \mathrm{C}$ constant temperature (Dubin et al., 2015). For mutants, total RNA was extracted from aerial parts of nine-leaf stage seedlings collected at $8 \mathrm{~h}$ after dawn using 
RNeasy mini kit (Qiagen) with DNase treatment (Thermo Fisher Scientific). cDNA was synthesized using the SuperScript III First-Strand Synthesis System (Invitrogen). qRT-PCR was performed using the LightCycler 96 system (Roche) with FastStart Essential DNA Green Master (Roche). SAND (AT2G28390) was used for a control to normalize the transcript abundance (Czechowski et al., 2005) using the ddCT method. The primer's sequences were SAND: 5'-AACTCTATGCAGCATTTGATCCACT-3' and 5'-TGATTGCATATCTTTATCGCCATC-3', and FLC: 5'-TGAGAACAAAAGTAGCCGACAAG-3' and 5'-ATGCGTCACAGAGAACAGAAAGC-3'.

\section{Assessment of AOP2's functionality}

\section{Tissue disruption}

Frozen $10 \mathrm{mg}$ samples in liquid nitrogen in $2 \mathrm{ml}$ Eppendorf tubes were stored at $-80{ }^{\circ} \mathrm{C}$. Precooled $1 \mathrm{ml} 90 \%$ methanol $\left(90 \% \mathrm{MeOH} / 10 \% 10 \mathrm{mM}\right.$ ammonium bicarbonate in $\mathrm{H}_{2} \mathrm{O} ;-20$ ${ }^{\circ} \mathrm{C}$ ) was added by the final methanol concentration remaining above $78 \%$. Tissue was disrupted by adding two small stainless beads bearings and agitating with a tissue lyser (tissuelyser II, Qiagen, Hilden, Germany) for $10 \mathrm{~min}$ at $20 \mathrm{rev} / \mathrm{s}$. The sample was shaken for a further $60 \mathrm{~min}(70 \mathrm{rev} / \mathrm{s})$ in the cold. After centrifuge at 13,000 rpm and the supernatant was transferred to a fresh tube and discard the pellet.

\section{Measurement}

Measurement of glucosinolates were performed according to a previous study (Liang et al., 2018). Briefly, $2 \mu \mathrm{l}$ of each sample was injected into a SeQuant ZIC-pHILIC HPLC column (Merck, $100 \times 2.1 \mathrm{~mm} ; 5 \mu \mathrm{m}$ ), and the respective guard column operated with an Ultimate 3000 HPLC system (Dionex, Thermo Fisher Scientific) at a flow rate of $100 \mu \mathrm{l} / \mathrm{min}$. The HPLC was directly coupled via electrospray ionization in the negative ion mode ( $2.8 \mathrm{kV})$ to a TSQ Quantiva mass spectrometer (Thermo Fisher Scientific). A linear gradient (A: 95\% acetonitrile $5 \%, 10 \mathrm{mM}$ aqueous ammonium acetate; $\mathrm{B}: 5 \mathrm{mM}$ aqueous ammonium acetate) starting with $5 \% \mathrm{~B}$ and ramping up to $45 \% \mathrm{~B}$ in 9 minutes was used for separation. Chromatograms were interpreted using TraceFinder (Thermo Fisher Scientific) and manually validated. The following transitions were used for relative quantitation: 3-hydroxypropyl glucosinolate $\mathrm{m} / \mathrm{z} 376 \rightarrow \mathrm{m} / \mathrm{z} 97 ; \mathrm{m} / \mathrm{z} 376 \rightarrow \mathrm{m} / \mathrm{z}$ 259, 2-propenyl glucosinolate $\mathrm{m} / \mathrm{z} 358 \rightarrow$ $\mathrm{m} / \mathrm{z}$ 97; m/z $358 \rightarrow \mathrm{m} / \mathrm{z}$ 75, 4-hydroxybutyl glucosinolate $\mathrm{m} / \mathrm{z} 390 \rightarrow \mathrm{m} / \mathrm{z} 259 ; \mathrm{m} / \mathrm{z} 390 \rightarrow$ $\mathrm{m} / \mathrm{z} 97 ; \mathrm{m} / \mathrm{z} 390 \rightarrow \mathrm{m} / \mathrm{z} 75$ and 3-(methylsulfinyl)propyl glucosinolate $\mathrm{m} / \mathrm{z} 422 \rightarrow \mathrm{m} / \mathrm{z} 259$; $\mathrm{m} / \mathrm{z} 422 \rightarrow \mathrm{m} / \mathrm{z} 97 ; \mathrm{m} / \mathrm{z} 422 \rightarrow \mathrm{m} / \mathrm{z} 75$.

\section{Acknowledgments}

The authors would like to thank Caroline Dean for sharing knowledge and seeds of the AtNDX1 mutant. We also thank Ümit Selen for technical support with the data analysis, and Daniel J. Kliebenstein and Haijun Liu for comments and helpful discussions. The VBCF Metabolomics Facility is supported by the City of Vienna through the Vienna Business Agency.

\section{Conflict of Interest}

The authors have no conflict of interest to declare. 


\section{References}

1001 Genomes Consortium (2016). 1,135 Genomes Reveal the Global Pattern of Polymorphism in Arabidopsis thaliana. Cell 166: 481-491.

Andrés F, Coupland G (2012). The genetic basis of flowering responses to seasonal cues. Nature Reviews Genetics 13: 627-639.

Atwell S, Huang YS, Vilhjálmsson BJ, Willems G, Horton M, Li Y, et al. (2010). Genome-wide association study of 107 phenotypes in Arabidopsis thaliana inbred lines. Nature 465: 627-631.

Blazquez MA, Green R, Nilsson O, Sussman MR, Weigel D (1998). Gibberellins promote flowering of arabidopsis by activating the LEAFY promoter. Plant Cell 10: 791-800.

Boyle EA, Li YI, Pritchard JK (2017). An Expanded View of Complex Traits: From Polygenic to Omnigenic. Cell 169: 1177-1186.

Brachi B, Faure N, Horton M, Flahauw E, Vazquez A, Nordborg M, et al. (2010). Linkage and association mapping of Arabidopsis thaliana flowering time in nature. PLoS Genet 6 : e1000940.

Chong VK, Stinchcombe JR (2019). Evaluating Population Genomic Candidate Genes Underlying Flowering Time in Arabidopsis thaliana Using T-DNA Insertion Lines. $J$ Hered 110: 445-454.

Czechowski T, Stitt M, Altmann T, Udvardi MK, Scheible W-R (2005). Genome-Wide Identification and Testing of Superior Reference Genes for Transcript Normalization in Arabidopsis. Plant Physiol 139: 5-17.

Dickson SP, Wang K, Krantz I, Hakonarson H, Goldstein DB (2010). Rare variants create synthetic genome-wide associations. PLoS Biol 8: e1000294.

Dubin MJ, Zhang P, Meng D, Remigereau M-S, Osborne EJ, Paolo Casale F, et al. (2015). DNA methylation in Arabidopsis has a genetic basis and shows evidence of local adaptation. Elife 4: e05255.

Flint J, Eskin E (2012). Genome-wide association studies in mice. Nat Rev Genet 13: 807-817.

Haley CS, Knott SA (1992). A simple regression method for mapping quantitative trait loci in line crosses using flanking markers. Heredity 69: 315-324.

Hormozdiari F, Kostem E, Kang EY, Pasaniuc B, Eskin E (2014). Identifying causal variants at loci with multiple signals of association. Genetics 198: 497-508.

Horton MW, Hancock AM, Huang YS, Toomajian C, Atwell S, Auton A, et al. (2012). Genome-wide patterns of genetic variation in worldwide Arabidopsis thaliana accessions from the RegMap panel. Nat Genet 44: 212-216.

Huang X, Wei X, Sang T, Zhao Q, Feng Q, Zhao Y, et al. (2010). Genome-wide association 
studies of 14 agronomic traits in rice landraces. Nat Genet 42: 961-967.

Jensen LM, Jepsen HSK, Halkier BA, Kliebenstein DJ, Burow M (2015). Natural variation in cross-talk between glucosinolates and onset of flowering in Arabidopsis. Front Plant Sci 6: 697.

Johanson U, West J, Lister C, Michaels S, Amasino R, Dean C (2000). Molecular analysis of FRIGIDA, a major determinant of natural variation in Arabidopsis flowering time.

Science 290: 344-347.

Kang HM, Zaitlen NA, Wade CM, Kirby A, Heckerman D, Daly MJ, et al. (2008). Efficient control of population structure in model organism association mapping. Genetics 178: 1709-1723.

Katz E, Bagaza C, Holden S, Angelovici R, Kliebenstein DJ (2020). Genetic variation, environment and demography intersect to shape Arabidopsis defense metabolite variation across Europe. Cold Spring Harbor Laboratory: 2020.09.23.310268.

Kerdaffrec E, Filiault DL, Korte A, Sasaki E, Nizhynska V, Seren Ü, et al. (2016). Multiple alleles at a single locus control seed dormancy in Swedish Arabidopsis. Elife $\mathbf{5}$.

Kerwin RE, Jimenez-Gomez JM, Fulop D, Harmer SL, Maloof JN, Kliebenstein DJ (2011). Network quantitative trait loci mapping of circadian clock outputs identifies metabolic pathway-to-clock linkages in Arabidopsis. Plant Cell 23: 471-485.

Kliebenstein DJ, Lambrix VM, Reichelt M, Gershenzon J, Mitchell-Olds T (2001). Gene Duplication in the Diversification of Secondary Metabolism: Tandem 2-Oxoglutarate-Dependent Dioxygenases Control Glucosinolate Biosynthesis in Arabidopsis. Plant Cell 13: 681-693.

Koornneef M, Alonso-Blanco C, Peeters AJM, Soppe W (1998). Genetic control of flowering time in Arabidopsis. Annu Rev Plant Physiol Plant Mol Biol 49: 345-370.

Larsson SJ, Lipka AE, Buckler ES (2013). Lessons from Dwarf8 on the strengths and weaknesses of structured association mapping. PLoS Genet 9: e1003246.

Liang X, Lee HW, Li Z, Lu Y, Zou L, Ong CN (2018). Simultaneous Quantification of 22 Glucosinolates in 12 Brassicaceae Vegetables by Hydrophilic Interaction Chromatography-Tandem Mass Spectrometry. ACS Omega 3: 15546-15553.

Li P, Filiault D, Box MS, Kerdaffrec E, van Oosterhout C, Wilczek AM, et al. (2014). Multiple FLC haplotypes defined by independent cis-regulatory variation underpin life history diversity in Arabidopsis thaliana. Genes Dev 28: 1635-1640.

Li Y, Huang Y, Bergelson J, Nordborg M, Borevitz JO (2010). Association mapping of local climate-sensitive quantitative trait loci in Arabidopsis thaliana. Proc Natl Acad Sci U S A 107: 21199-21204.

Lippert C, Casale FP, Rakitsch B, Stegle O (2014). LIMIX: genetic analysis of multiple traits. bioRxiv.

Long Q, Rabanal FA, Meng D, Huber CD, Farlow A, Platzer A, et al. (2013). Massive genomic variation and strong selection in Arabidopsis thaliana lines from Sweden. Nat 
Genet 45: 884-890.

MacArthur J, Bowler E, Cerezo M, Gil L, Hall P, Hastings E, et al. (2017). The new NHGRI-EBI Catalog of published genome-wide association studies (GWAS Catalog). Nucleic Acids Res 45: D896-D901.

Martínez O, Curnow RN (1992). Estimating the locations and the sizes of the effects of quantitative trait loci using flanking markers. Theor Appl Genet 85: 480-488.

Maynard Smith J, Haigh J (1974). The hitch-hiking effect of a favourable gene. Genet Res 23: 23-35.

Neal CS, Fredericks DP, Griffiths CA, Neale AD (2010). The characterisation of AOP2: a gene associated with the biosynthesis of aliphatic alkenyl glucosinolates in Arabidopsis thaliana. BMC Plant Biol 10: 170.

Platt A, Vilhjálmsson BJ, Nordborg M (2010). Conditions Under Which Genome-Wide Association Studies Will be Positively Misleading. Genetics 186: 1045-1052.

Porri A, Torti S, Romera-Branchat M, Coupland G (2012). Spatially distinct regulatory roles for gibberellins in the promotion of flowering of Arabidopsis under long photoperiods. Development 139: 2198-2209.

Reeves PH, Coupland G (2001). Analysis of flowering time control in Arabidopsis by comparison of double and triple mutants. Plant Physiol 126: 1085-1091.

Sasaki E, Frommlet F, Nordborg M (2017). The genetic architecture of the network underlying flowering time variation in Arabidopsis thaliana. : 175430.

Sasaki E, Zhang P, Atwell S, Meng D, Nordborg M (2015). ' Missing' G x E variation controls flowering time in Arabidopsis thaliana. PLoS Genet 11: e1005597.

Scheet P, Stephens M (2006). A fast and flexible statistical model for large-scale population genotype data: applications to inferring missing genotypes and haplotypic phase. $A m \mathrm{~J}$ Hum Genet 78: 629-644.

Seren Ü, Grimm D, Fitz J, Weigel D, Nordborg M, Borgwardt K, et al. (2017). AraPheno: a public database for Arabidopsis thaliana phenotypes. Nucleic Acids Res 45: D1054-D1059.

Srikanth A, Schmid M (2011). Regulation of flowering time: all roads lead to Rome. Cellular and Molecular Life Sciences 68: 2013-2037.

Sun Q, Csorba T, Skourti-Stathaki K, Proudfoot NJ, Dean C (2013). R-loop stabilization represses antisense transcription at the Arabidopsis FLC locus. Science 340: 619-621.

Sun TP, Kamiya Y (1994). The Arabidopsis GA1 locus encodes the cyclase ent-kaurene synthetase A of gibberellin biosynthesis. Plant Cell 6: 1509-1518.

Tam V, Patel N, Turcotte M, Bossé Y, Paré G, Meyre D (2019). Benefits and limitations of genome-wide association studies. Nat Rev Genet 20: 467-484.

Vilhjálmsson BJ, Nordborg M (2013). The nature of confounding in genome-wide association 
studies. Nat Rev Genet 14: 1-2.

Whittaker C, Dean C (2017). The FLC Locus: A Platform for Discoveries in Epigenetics and Adaptation. Annu Rev Cell Dev Biol 33: 555-575.

Yu J, Pressoir G, Briggs WH, Vroh Bi I, Yamasaki M, Doebley JF, et al. (2006). A unified mixed-model method for association mapping that accounts for multiple levels of relatedness. Nat Genet 38: 203-208.

Zan Y, Carlborg Ö (2019). A Polygenic Genetic Architecture of Flowering Time in the Worldwide Arabidopsis thaliana Population. Mol Biol Evol 36: 141-154. 